\title{
BMJ Open Formalisation and subordination: a contingency theory approach to optimising primary care teams
}

\author{
Damien Contandriopoulos, ${ }^{1}$ Mélanie Perroux, ${ }^{2}$ Arnaud Duhoux ${ }^{3}$
}

To cite: Contandriopoulos D, Perroux M, Duhoux A. Formalisation and subordination: a contingency theory approach to optimising primary care teams. BMJ Open 2018;8:e25007. doi:10.1136/ bmjopen-2018-025007

- Prepublication history for this paper is available online. To view these files, please visit the journal online (http://dx.doi. org/10.1136/bmjopen-2018025007).

Received 4 July 2018 Revised 19 October 2018 Accepted 25 October 2018
Check for updates

(c) Author(s) (or their employer(s)) 2018. Re-use permitted under CC BY-NC. No commercial re-use. See rights and permissions. Published by BMJ.

${ }^{1}$ School of Nursing, University of Victoria, Victoria, British Columbia, Canada

${ }^{2}$ Regroupement des Aidants Naturels du Québec, Montreal, Canada

${ }^{3}$ Université de Montréal,

Montreal, Canada

Correspondence to

Professor Damien

Contandriopoulos;

damien1@uvic.ca

\section{ABSTRACT}

Objective While there is consensus on the need to strengthen primary care capacities to improve healthcare systems' performance and sustainability, there is only limited evidence on the best way to organise primary care teams. In this article, we use a conceptual framework derived from contingency theory to analyse the structures and process optimisation of multiprofessional primary care teams.

Design We focus specifically on inter-relationships between three dimensions: team size, formalisation of care processes and nurse autonomy. Interview-based qualitative data for each of these three dimensions were converted into ordinal scores. Data came from eight pilot sites in Quebec (Canada).

Results We found a positive association between team size and formalisation (correlation score 0.55 ) and a negative covariation (correlation score -0.64 ) between care process formalisation and nurses' autonomy/ subordination. Despite the study being exploratory in nature, such relationships validate the idea that these dimensions should be analysed conjointly and are coherent with our suggestion that using a framework derived from a contingency approach makes sense.

Conclusions The results provide insights about the structural design of nurse-intensive primary care teams. Non-physicians' professional autonomy is likely to be higher in smaller teams. Likewise, a primary care team that aims to increase nurses' and other non-physicians' professional autonomy should be careful about the extent to which it formalises its processes.

\section{INTRODUCTION}

The continued reliance on current healthcare provision models to address evolving population health needs is likely to exert considerable pressure on public finances. ${ }^{1}$ Likewise, technical innovations and rapid growth in the intensity of care being provided will exacerbate the issue. ${ }^{23}$ Available evidence, thus, suggests that public health systems need to change significantly to preserve their capacity to maintain universal access to healthcare. ${ }^{45}$

Strengthening primary care capacities is widely considered to be an approach with the potential to reinforce simultaneously health system sustainability and accessibility,

\section{Strengths and limitations of this study}

This article relies on contingency theory to analyse how multiprofessional primary care teams are optimised.

- Qualitative data from eight primary care pilot sites were used to assess the covariation between ordinal estimates of team size, formalisation of care processes and nurse autonomy.

- The analysis was exploratory and based on correlation analysis using a convenience sample.

continuity of care, and ultimately, population health. ${ }^{35-11}$ However, for this to happen, available evidence suggests that efforts should not be limited to funding more of the same. ${ }^{12}$ Primary care strengthening should include redefining the nature of the care provided as well as the professional roles and task sharing within teams. ${ }^{73-16}$ This article analyses factors affecting the functioning of interprofessional teams in order to support the strengthening of primary care delivery structures and processes.

From a narrative review of the literature, ${ }^{12}$ we identified two important dimensions to consider in classifying and analysing promising multiprofessional primary care teams. One is the degree to which the division of tasks in the team is formalised, and the other is whether the core professional around whom the practice is structured is a physician or an advanced practice nurse.

Building on these dimensions, we argue here that a conceptual lens derived from contingency theory can be helpful to understand how best to optimise the structure and processes of multiprofessional primary care teams. We first discuss the theoretical underpinnings of such a view, and then we present an empirical analysis of this relationship using data from eight primary care teams studied in the context of a broader project in Quebec. ${ }^{17}$ 


\section{CONCEPTUAL FRAMEWORK}

Contingency theory is the dominant school of thought for analysing the link between organisations' functioning and performance. ${ }^{18}$ This theory's core idea is that there is no one best way. Performance is not a product of organisational structure or processes per se. Performance needs to be conceived as a product of the fit between the organisation's functioning and a set of contingency factors such as organisational size, age and environmental predictability.

Our analysis is not focused on organisational performance, but relies on a similar logic to analyse the optimisation of professional roles in primary care teams. We contend there is likely no one best way to organise primary care teams for dimensions such as professional roles definition, task sharing and team size.

Our interest in applying a contingency approach to role definition stems in part from the results of a narrative review we conducted of the literature on high-performance nurse-intensive primary care models. ${ }^{12}$ The review revealed various plausible operationalisations of high-performance multiprofessional primary care teams-not one best way, but different coherent articulations of the resources at hand, given environmental constraints and team objectives. The broad range of organisational structures that, according to the literature, appears to succeed in delivering high-quality care efficiently and effectively points to the need for a contingency approach to understand primary care structures and process optimisation.

The review also led to the identification of two dimensions that appeared important for classifying and analysing promising multiprofessional primary care teams. The first was the degree to which the division of tasks in the team was formalised. At one extreme were formal models relying on explicit procedures that specified what service would be provided by which professional, to which patient, and at what point in time. At the other were organic approaches based on mutual adjustments, in which professionals adapted to structural circumstances and to patients' characteristics in deciding on care processes. ${ }^{19}$

The second dimension was the training of the core professional around whom the practice was structured. Most primary care models are physician centred, but there is a growing number of nurse-centred teams. When a model is nurse centric, the core position is usually occupied by a primary healthcare nurse practitioner (NP) (NPs' training and legal scope of practice varies from one jurisdiction to the next. We use the term here to refer to nurses with graduate level university training and an extended scope of practice, including some prescribing rights, such that they are allowed to diagnose autonomously and treat a variety of common conditions). The relative centrality of either physicians or nurses and the level of autonomy granted to non-physician professionals is a second structuring dimension for the classification of primary care teams.
In our review, these two dimensions were key parameters to classify and describe multiprofessional primary care teams but the literature we analysed did not posit any interdependency between the two. However, as we discuss below, the two dimensions of formalisation and professional role relate to elements that are abundantly discussed in the literature on healthcare organisations.

\section{Professionalism, formalisation and primary care delivery}

From its inception, the organisation science literature has strongly advocated the virtues of formalised and standardised production processes, ${ }^{20}{ }^{21}$ the ultimate form of which would become the production line. However, despite the impressive successes of this approach in some industries, such as large-scale manufacturing, it failed to generalise to all sectors. Of particular interest here, organisations in which humans are the raw material ${ }^{22}$ rely on processes that are not easily standardised. Those organisations (healthcare, education, social services, etc) thus tend to rely on a highly trained and specialised workforce responsible for handling the inherent unpredictability of the production. ${ }^{23}$

In the late 1970s, Mintzberg deeply influenced the field by synthesising contingency theory findings into a configurational approach. ${ }^{24} \mathrm{He}$ offered five ideal types of potential fit between well-documented contingency factors and organisational structures. One ideal type, the professional bureaucracy, was coined to describe organisations that, on one hand, are forced to decentralise the control of production processes to relatively autonomous professionals and, on the other, rely on formalisation and standardisation for all the predictable portions of their operations. The poster child of a professional bureaucracy is the acute care hospital.

Because of their organisational simplicity and small size, other care provision structures such as private clinics, physicians' offices and the like were usually described as 'simple structures' in Mintzberg's terms. In those, a few professionals would rely on self-adjustment and direct control to run the operations. However, today the independent small-scale physician's office is a near-extinct species as a care delivery model. Primary care production is increasingly in the hands of larger, multiprofessional structures ${ }^{7}$ dealing with many of the characteristic contingencies of professional bureaucracies (eg, size, age, vertical integration, division of labour among professionals and unpredictable production processes)..$^{25}$

Given the current evolution in primary care production structures, we believe a conceptual lens derived from contingency theory might help deepen our understanding of their optimal functioning parameters. Moreover, building on results from the above-mentioned narrative review, ${ }^{12}$ we will focus mainly on formalisation and professional autonomy as the two core contingency parameters to be considered. 


\section{Defining formalisation}

Formalisation can be conceived as both a process and an outcome. As an outcome, it is the extent to which behaviour within a team is prescribed by explicit procedures and rules. ${ }^{26} 27$ This has to do with what Dalton et $a l^{28}$ call the structuring-as opposed to the structural-components of organisational structure. ${ }^{21}$ Structural components describe the arrangements (such as size, subunit sizes, span of control, etc) through which participants are formally interconnected. Structuring components are more processual in nature and refer to elements such as policies and activities aimed at codifying the behaviour of participants. As a process, formalisation is therefore defined as efforts to increase the structuring of behaviours in order to strengthen the predictability of actions and decrease role ambiguity. ${ }^{29}$ This definition emphasises the existence of a deliberate intention, whether of a person or a group, to increase predictability. Formalisation conceived as a process is also logically linked to its conception as an outcome. Procedures aimed at increasing behaviour predictability are likely to lead to given behaviours. We do acknowledge that strong macrosystemic, non-deliberate formalisation forces exist that exert influence through the social processes described, for example, in the neoinstitutional organisational literature. ${ }^{30-32}$ Our emphasis here on deliberate interventions was adopted because of the specific objectives of the study and not the relative importance of the micro/macroformalisation pressures.

\section{Defining professional autonomy and subordination}

As stated earlier, the results from the narrative review suggest that two professional groups are commonly found at the centre of any primary care delivery model: physicians and nurses. However, despite its seemingly dichotomous nature, we argue this characteristic of primary care models should be conceived on a continuum of professional autonomy and subordination. ${ }^{33}$

Historically, the medical profession has been very successful at claiming and protecting a monopoly over the provision of most human healthcare. However, to deliver the care while relying on a relatively scarce workforce, the medical profession has delegated a significant portion of the day-to-day work to 'subordinate' professional groups, among which nursing is the most important. ${ }^{34}$ For members of those historically subordinated professions, practical professional autonomy is highly dependent on the level of subordination of processes in their work environment.

Nurses are the most commonly found non-physician professionals in primary care and play a significant role in almost all primary care models. In teams where the core professional is a physician, nurses' level of professional autonomy varies greatly. At one end of the spectrum are physician-centric models in which nurses are highly subordinated, with a limited scope of practice and little autonomy. At the other end are nurse-centric models, such as nurse-led clinics, like those existing in Ontario or in the USA, ${ }^{35-37}$ where nurses have almost complete autonomy. In between lies a vast array of configurations, including interprofessional teams in which nurses enjoy varying levels of autonomy regarding the care they provide to their patients.

\section{DATA AND METHODS}

The results presented here are part of a larger project whose complete research protocol is described in Contandriopoulos et $a l_{.}{ }^{17}$ The general objective of that project is to understand the characteristics of high-performance primary care teams and to assess their outcomes through a two components mixed-method study design. The data used here were derived from the qualitative first component, which was an implementation analysis based on developmental evaluation principles. ${ }^{38} 39$

\section{Data}

The eight primary care teams included in the study are all located in Quebec (Canada) and within either urban (Montreal or Quebec) or dense suburban settings (Montreal South-shore). They were selected on the basis of preliminary analysis showing they were all highly interprofessional (at least half of the professional workforce is composed of non-physicians $)^{16}$ and likely characterised by high levels of effectiveness and efficiency (according to the opinion of regional and provincial experts collected during preselection interviews). The sample is thus deliberately not representative of the average primary care team in Quebec. The sampling logic is in line with the goal of the study, which is to understand the characteristics of high-performance primary care teams.

In each of the eight primary care settings, semistructured interviews were conducted with various informants (physicians, nurses, administrators, etc) to identify the characteristics of the care structures and processes. The conceptual framework used for this is available in Contandriopoulos $e \mathrm{al}^{12}{ }^{12}$ A total of 73 interviews were conducted with a total of 53 informants (15 physicians, 9 NPs, 18 registered nurses and licensed practical nurses, 4 administrators, 8 other professionals such as social workers and psychologists). Interviews lasted between $45 \mathrm{~min}$ and 2 hours and were conducted by experienced researchers. The focus of the interviews was on the evolution of the practice model, the way professional roles were structured and evolved, the ways in which professional roles and patient flow interact, professional communication processes, administrative and decision-making processes. Non-participant observation was also conducted in most settings to document team dynamics and organisational culture.

\section{Patient and public involvement}

There was no direct patient or public involvement in the research component whose results are discussed here. However, this component is part of a larger project in which 3000 patients and user are being followed 
longitudinally to analyse the evolution of their satisfaction regarding services and their perception of unmet needs in the eight pilot sites.

\section{Measuring formalisation and autonomy}

We found no scales of formalisation or autonomy that could be applied directly to our object. In both cases, we relied on adaptations of existing tools.

The starting point of the scale we used to measure formalisation is based on the seminal work of Hall $e t a l^{25}$ which provides five dimensions, each appraised through two or three criteria (11 in total). ${ }^{18}$ Hall typology relies on the causal assumption that rules and procedures aimed at increasing behaviour predictability are a good proxy of actual human processes and therefore a predictor of formalisation outcomes. To use this scale in our study, we had to edit it in two ways. First, we edited the criteria to make them more primary care specific. Second, we edited some items to remove conceptual overlap with the notion of subordination. For example, in the context of primary care teams, the existence of a 'clear definition of the hierarchy of authority', as per Hall $e t a l^{25}$ would be largely overlapping with the concept of physician subordination as defined earlier. As we discuss below, the data used here were derived from in-depth qualitative interviews. Some elements of Hall $e t a t^{25}$ typology were not documented in the interviews and therefore were not included. Table 1 presents the selected criteria from Hall $e t a l^{25}$ and the way we operationalised them.

For the autonomy/subordination scale, we mostly relied on the work of Adamson $e t a l^{40}$ and Hojat $e t a l^{41} \mathrm{In}$ those two scales, we selected items based on two criteria. First, an item had to have obvious conceptual ties with the concept of subordination in primary healthcare delivery. Second, the item had to be focused on actual practices or processes rather than on perceptions. Table 2 presents the selected criteria from Adamson et $a l^{40}$ and Hojat, et $a t^{41}$ and how we operationalised them.

\section{Score attribution}

Interviews were recorded and transcribed and then analysed both by site and cross-sectionally. The analysis was based on discourse analysis techniques. ${ }^{42-46}$ The end product was an in-depth narrative profile of each site. Draft versions of each profile were discussed at research team meetings and adjusted until they were perceived as being both accurate in relation to the interview transcripts and comprehensive in regard to available information. At that point, profiles were sent to each informant for validation and then further edited based on their feedback.

For the purpose of the present analysis, preliminary scores for each criterion of the formalisation and autonomy/subordination scales were established independently by two researchers based on each site's profile. Every criterion (see tables 1 and 2) is phrased as a descriptive claim regarding observable characteristics of the team. Ordinal scores of either 0,1 or2 were attributed depending on the claim being true, sometimes true or
Table 1 Operationalisation of the measure of formalisation

Operationalisation in this

Hall et al 1967 typology study

A. Roles
The degree to which the Non-physicians' professional positions in the organisation roles are divided according to are concretely defined.

'care modules' mostly based on diseases. For example, diabetes, mental health, (yes=2, to some extent $=1$, no=0).

\begin{tabular}{|c|c|}
\hline \multicolumn{2}{|l|}{ B. Authority relations } \\
\hline $\begin{array}{l}\text { The degree to which the } \\
\text { authority structure is } \\
\text { formalised (clear definition of } \\
\text { the hierarchy of authority). }\end{array}$ & $\begin{array}{l}\text { There are formal rules that } \\
\text { specify which patients will be } \\
\text { treated by which professional } \\
\text { (yes }=2 \text {, to some extent }=1 \text {, } \\
\text { no=0). }\end{array}$ \\
\hline
\end{tabular}

\section{Communications}

The degree of emphasis on written communications.

An electronic health record (EHR) system is used to communicate patient information between professionals within the team (yes=2, EHR exists but is not the main communication tool $=1$, no=0).

$\begin{array}{ll}\begin{array}{l}\text { The degree of emphasis } \\ \text { ongoing through }\end{array} & \begin{array}{l}\text { Professionals will have team } \\ \text { discussions on complex } \\ \text { established channels in the } \\ \text { patients (systematically=2, } \\ \text { if needed =1, informal chats } \\ \text { only=0). }\end{array}\end{array}$

D. Norms and sanctions

The no of written rules and
policies. $\begin{aligned} & \text { Collective prescription rules } \\ & \text { are in place to structure } \\ & \text { non-physicians' capacity to } \\ & \text { provide drugs to patients } \\ & \text { they treat (yes=2, some=1, } \\ & \text { none=0). }\end{aligned}$

\section{E. Procedures}

The degree of formalisation Work within the team is of orientation programmes structured according to for new members (systematic formal teamlets (yes=2, yes, socialisation for all new entrants). but with flexibility $=1, \mathrm{no}=0$ ).

false. Those preliminary scores were then discussed in team meetings involving the researchers who conducted the interviews. There was an initial discrepancy in 6 of the 132 items (4.5\%). As scores were based on descriptive claims, the discrepancies were all resolved by identifying whether the interview data supported the claim or not.

However, those team discussions also made it clear that some larger primary care sites in our sample actually consisted of distinct submodels of practice. Specifically, in some settings, the practice model was different depending on whether the core professional was an NP or a physician. We, therefore, divided four of our sites 
Table 2 Operationalisation of the measure of nurses' autonomy versus subordination

\begin{tabular}{l} 
From Heinemann et $a^{53}$ \\
\hline The physician should not \\
always have the final word in \\
decisions made by healthcare \\
teams.
\end{tabular}

Operationalisation in this study

Nurses and other nonphysician professionals are involved in clinical decisions regarding their patients (yes, all the time $=2$, sometimes $=1$, rarely=0).

The physician has the ultimate legal responsibility for decisions made by healthcare teams.

\section{A physician has to be} physically present in the clinic at all times for services to be delivered ( $\mathrm{no}=2$, yes, but exceptions apply $=1$, yes $=0$ ).

$\begin{array}{ll}\text { From Hojat et al } 1999 & \begin{array}{l}\text { Operationalisation in this } \\ \text { study }\end{array}\end{array}$

Physicians and nurses should Nurses can treat and send a contribute to decisions patient back home without regarding the hospital asking permission from a discharge of patients. physician (yes $=2$, In some circumstances $=1$, no $=0$ ).

\begin{tabular}{ll}
$\begin{array}{l}\text { Nurses should be involved } \\
\text { in making policy decisions } \\
\text { concerning the hospital } \\
\text { support services on which } \\
\text { their work depends. }\end{array}$ & $\begin{array}{l}\text { Nurses and other non- } \\
\text { physician professionals are } \\
\text { involved in organisational/ } \\
\text { managerial decisions (yes, } \\
\text { all the time=2, sometimes=1, } \\
\text { rarely=0). }\end{array}$ \\
$\begin{array}{l}\text { The primary function of the } \\
\text { nurse is to carry out the } \\
\text { physician's orders. }\end{array}$ & $\begin{array}{l}\text { Nurses and other non- } \\
\text { physician professionals are } \\
\text { involved in supervision and } \\
\text { training activities (yes, all } \\
\text { the time=2, sometimes }=1, \\
\text { rarely=0). }\end{array}$ \\
\hline
\end{tabular}

into two subsites with different scores. This produced a total of 12 sites or subsites. For confidentiality purposes, sites were identified only through a two-letter code. Sites that were subdivided have either an-MD or-NP after their code names.

Having divided primary care sites according to such logic, it followed that what was meaningful for assessing the functioning of the team was not the formal organisational boundaries. First, Quebec's care delivery organisations have, in recent years, experienced large forced mergers, such that some of our study settings are now part of huge structures that include acute care hospitals, long-term care facilities and others. Obviously, those formal boundaries do not constitute a coherent measure of primary care team size. Second, according to the headcounts in the various physical locations, some of our study settings are quite large, with a total workforce of over 100 persons, while others have less than 10 . In larger teams' daily work, the professionals function within more circumscribed work environments. Nevertheless, being a small subteam within a large structure necessitates coordination mechanisms with the rest of the organisation that a small-scale practice does not require. This is contingent, however, on the actual level of interdependence of the organisational components, which in turn depends on the practice model. For these reasons, properly assessing team size was not as straightforward as might have been expected.

Based on the elements discussed above, we estimated practical team size based on three factors: size of the overall organisation $(0-10$ full time equivalent $(\mathrm{FTE})=1$; 20-50 $\mathrm{FTE}=2$; 50 and more $\mathrm{FTE}=3)$; size of the care delivery site (0-5 FTE=1; 5-15 FTE=2; 15 and more FTE $=3$ ) and level of interdependency in daily practice within the team (minimal=1; moderate=2; high=3). Size scores were established and validated according to the same approach described earlier. Initial discrepancies in 3 items out of $36(8.3 \%)$, all related to the interdependency dimension, were resolved by identifying whether interview data supported the claim or not.

\section{Analysis}

Based on the conceptual framework presented earlier, we sequentially assessed the covariation between size and formalisation and between formalisation and autonomy. For each three dimensions, we relied on site total scores based on the sum of each criterion for each dimension.

Given the exploratory nature of this study and the data used, we limited ourselves to simple Pearson correlation coefficients. We conducted sensitivity analyses by removing settings with the highest and lowest scores. The covariation between the dimensions studied was also visually analysed on scatter graphs including a linear regression.

\section{RESULTS}

Table 3 presents the score for each criterion from the two scales for each site or subsite. The settings were sorted according to their formalisation scores.

We found a positive covariation between team size and formalisation (see figure 1). The Pearson correlation coefficient is 0.55 . Sensitivity tests show that the relation remains when the smallest site is removed $(0.30)$, when the largest site is removed (0.78) and when both are removed (0.63).

We also found a negative covariation between formalisation and autonomy (see figure 2). The Pearson correlation coefficient is -0.64 . We conducted sensitivity analyses by removing either the settings with the highest and lowest formalisation scores or those with the highest and lowest autonomy scores. In both cases the correlation decreased (respectively, to -0.26 and -0.47 ) but the direction of the covariation remained. Similarly, we tried merging back the primary care settings we had subdivided based on the training of the core professional by averaging their scores, and the same association remained (correlation score of -0.52). 


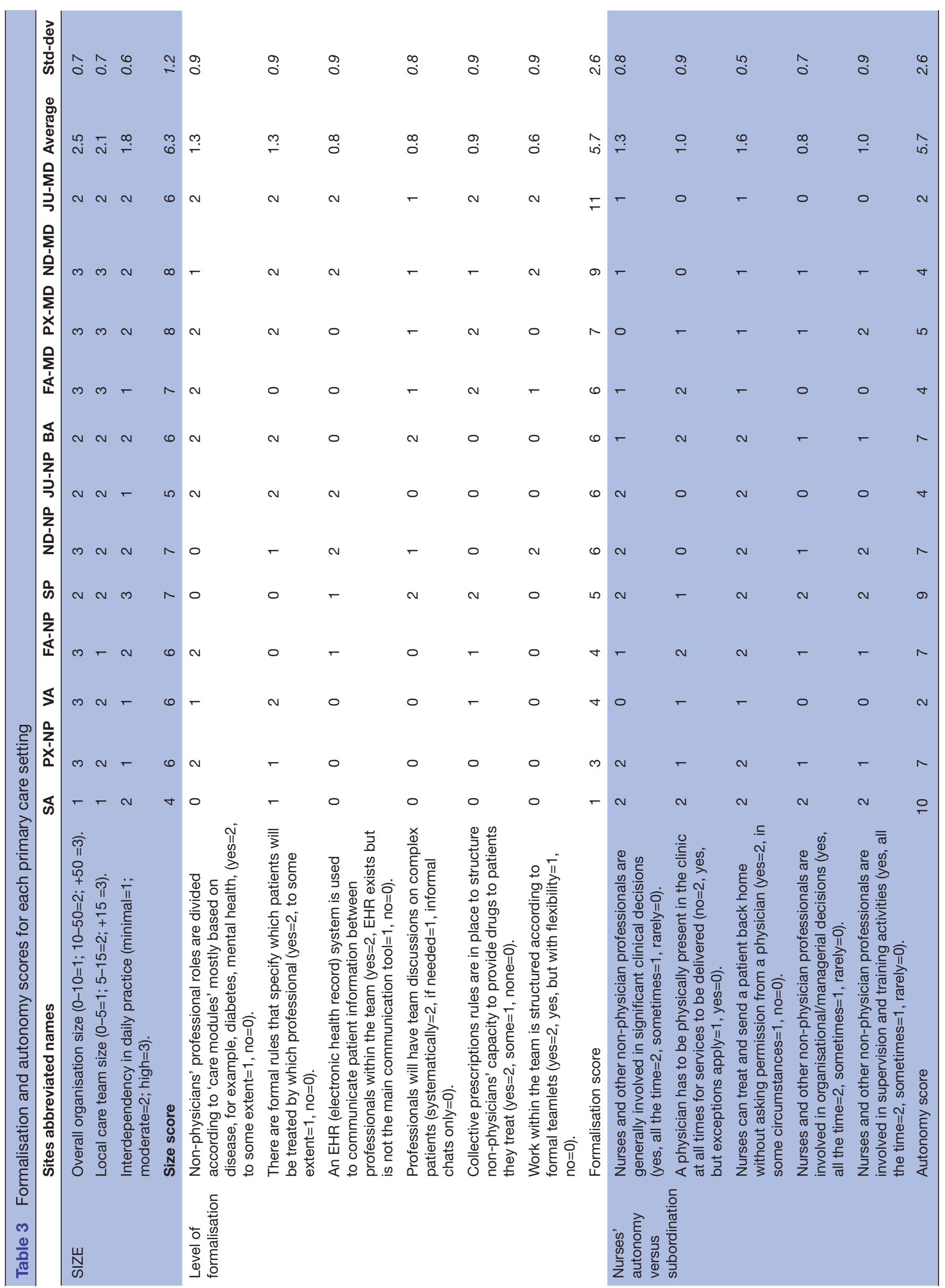




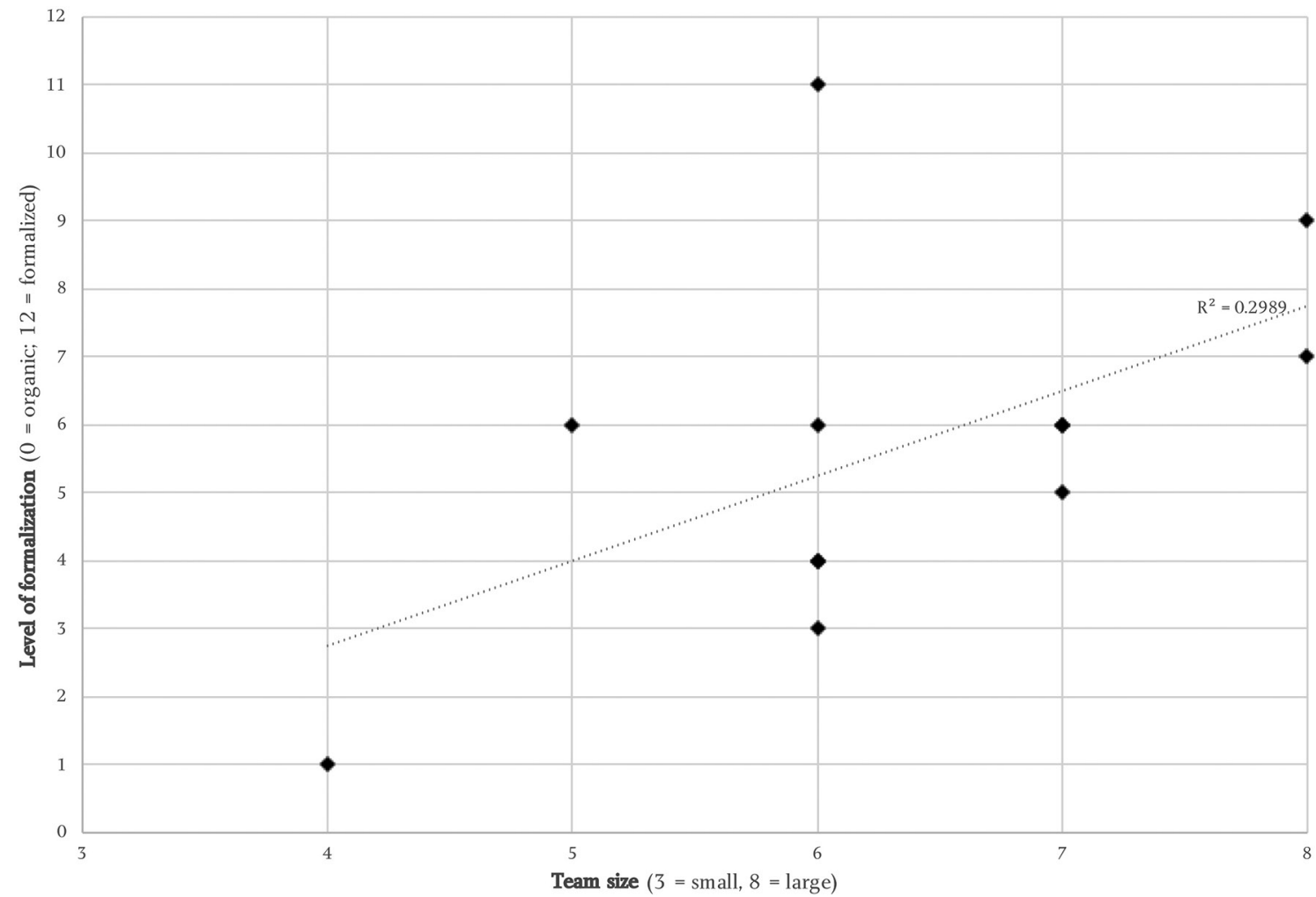

Figure 1 Relationship between team size and level of formalisation.

\section{DISCUSSION}

The results showed a negative covariation between the level of care process formalisation and the level of nurses' autonomy/subordination. Such a relationship validates the idea that those two dimensions should be analysed conjointly and is coherent with our suggestion that a conceptual framework inspired by a contingency approach makes sense.

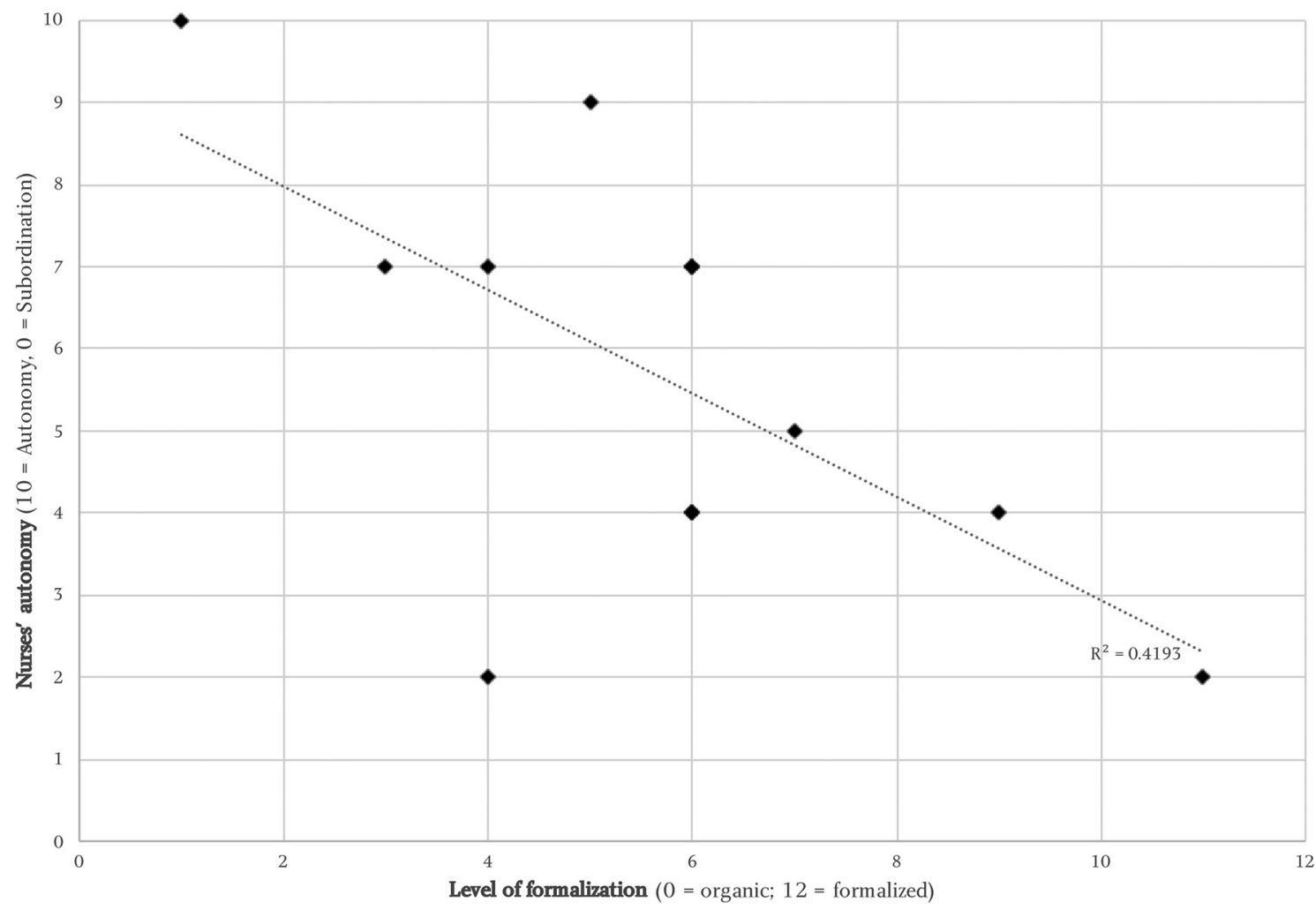

Figure 2 Relationship between formalisation and nurses' autonomy. 
The empirical data we used cannot inform on the plausibility of a causal relationship. However, conceptually, the hypothesis of a causal relationship in which an increased level of formalisation leads to a drop in nurses' autonomy has much face validity. As mentioned earlier, based on the literature, formalisation was defined as efforts made to strengthen the predictability of actions and decrease role ambiguity. We believe a partial explanation for the correlation found between the level of formalisation and nurses' professional autonomy is that efforts made by the teams to decrease role ambiguity tend to formalise roles in ways that restrict nurses' professional autonomy.

Our data also showed a great deal of variation in nurses' levels of autonomy for similar levels of formalisation. We interpret this variation as a sign that the relationship between formalisation and nurses' autonomy is far from direct and mechanical. For formalisation scores near the middle of the scale, no covariation existed with the level of nurses' autonomy. In-depth analysis of each practice setting also suggested teams have a great deal of agency in articulating their model of practice. We understand this as suggesting that, unless formalisation is pushed to extremes in any given direction, autonomy/subordination remains a dimension over which teams can have significant control.

However, according to a contingency theory approach, the optimal level of formalisation is not something organisations can fully decide on their own. There are parameters (eg, size, age, environmental predictability, managerial style, etc ${ }^{1824}$ ) that set a range of plausible levels. And indeed, the rough measure of organisational size we applied did correlate with our measure of formalisation. This suggests there would be some merit in conceptualising primary care teams' structural and process optimisation in a systemic way. The optimal choice for any given team is likely to be contingent on a set of interconnected parameters. For example, if team size is indeed positively associated with formalisation and, in turn, if high levels of formalisation tend to limit nurses' autonomy, then team size and nurse autonomy should be seen as interdependent. Such an observation is especially timely given that the current trend towards a more diverse and interprofessional workforce in primary care goes hand in hand with increasingly large practice size. ${ }^{7}$

Similarly, given the results obtained, we believe that if a primary care team aims to increase nurses' and other non-physicians' professional autonomy, it should be very careful about the extent to which it formalises its processes. This advice is also worth considering in relation to the avalanche of recommendations in the nursing literature suggesting that role clarification through formal definitions is the way forward to increase nurses' autonomy and scope of practice. ${ }^{48-50}$

\section{Limitations}

This study has a few limitations worth discussing. First, the analysis was based on secondary analysis of existing data. The hypothesis about a potential link between formalisation and autonomy emerged during the discussions about site descriptions. Therefore, the data collection was not initially aimed at measuring those dimensions. The data are nevertheless rich and detailed, and we are confident in their validity. However, the results presented here remain mostly exploratory, and further studies on the topic would be needed.

Second, our sample was limited to Quebec and deliberately skewed towards well-functioning teams whose care delivery processes relied to a large extent on non-physician professionals. It would be interesting to study the topic in other jurisdictions and with a more diverse sample of primary care teams, and especially teams with some variability in funding mechanisms. ${ }^{51} 52$

Finally, the third element of note here has to do with the nature of the data, but is not a limitation per se. Given the two limitations identified above, it would be tempting to measure formalisation and autonomy in a much larger sample of primary care teams using a quantitative survey instrument. However, we are not aware of any survey instrument that would provide a satisfying level of construct validity to be usable for such a purpose.

\section{CONCLUSION}

Our earlier narrative review of the literature showed that the level of formalisation and the training of the team's core professional are central parameters by which to classify and describe multiprofessional primary care teams. The exploratory analysis conducted here suggests those dimensions should be analysed together from a contingency perspective.

While most jurisdictions internationally try to identify and implement coherent and efficient ways to strengthen their primary care capacities, there is little evidence-informed advice in the literature about the parameters for doing so. The present study suggests that adopting a contingency perspective might be an interesting way to disentangle some parameters of primary care team optimisation. We also believe the covariations we found between team size, formalisation and nurses' autonomy could have a practical value for many teams currently grappling with the best way to rethink roles and processes.

Finally, at the time of writing this article, we do not have the data needed to assess whether there is a link between level of professional autonomy and a team's performance. On one hand, contingency theory suggests that under some circumstances it might make sense to restrict workers' autonomy. On the other, the consensus in most nurse-based literature is that increased nurse autonomy improves quality of care quality and efficiency. We believe this could be an interesting focus for further research.

\section{Original protocol for the study}

This study is part of a larger project whose detailed protocol was published and is in open access. ${ }^{17}$

Contributors MP had the initial idea to study the link between formalisation and autonomy and wrote the first drafts. DC developed the conceptual framework, 
analysed the data and wrote most of the paper. $A D$ was involved in the team discussion at all steps and contributed to the final paper.

Funding This work was supported by the Canadian Institutes for Health Research grant number MOP 136851. DC also benefits from a Canadian Institutes for Health Research applied public health research chair (grant number: CPP 137912).

Competing interests None declared.

Patient consent Not required.

Ethics approval The project, as well as all consent forms and research tools, was accepted by the University of Montreal Health Sciences Research Ethics Committee (CERES) and the Research Ethics Committee of the Centre de sante et de services sociaux de la Montagne.

Provenance and peer review Not commissioned; externally peer reviewed.

Data sharing statement The data used here are composed of interview verbatims that cannot be shared due to confidentiality issues.

Open access This is an open access article distributed in accordance with the Creative Commons Attribution Non Commercial (CC BY-NC 4.0) license, which permits others to distribute, remix, adapt, build upon this work non-commercially, and license their derivative works on different terms, provided the original work is properly cited, appropriate credit is given, any changes made indicated, and the use is non-commercial. See: http://creativecommons.org/licenses/by-nc/4.0/.

\section{REFERENCES}

1. Clavet NJ, Duclos JY, Fortin B, et al. Les dépenses en santé du gouvernement du Québec, 2013-2030: projections et déterminants. Montreal, QC: CIRANO Scientific Series, 2013. 2013s-45.

2. Commission on Social Determinants of Health. Achieving Health Equity: From Root Causes to Fair Outcomes. Interim Statement. Geneva. Geneva: World Health Organization, 2007.

3. Rittenhouse DR, Shortell SM, Fisher ES. Primary care and accountable care--two essential elements of delivery-system reform. N Engl J Med 2009;361:2301-3.

4. Casey D. Transforming the delivery of health and social care: the case for fundamental change. Br J Gen Pract 2013;63:292.1-292.

5. Starfield B, Shi L, Macinko J. Contribution of primary care to health systems and health. Milbank Q 2005;83:457-502.

6 Donaldson MS, Yordy KD, Lohr KN, et al. Primary Care: America's Health in a New Era (Committee on the Future of Primary Care, Institute of Medicine). Washington, DC: National Academies Press, 1996.

7. Bauer L, Bodenheimer T. Expanded roles of registered nurses in primary care delivery of the future. Nurs Outlook 2017;65:624-32.

8. Feachem RG, Sekhri NK, White KL, et al. Getting more for their dollar: a comparison of the NHS with California's Kaiser Permanente. BMJ 2002;324:135-43.

9. Haggerty JL, Pineault R, Beaulieu MD, et al. Practice features associated with patient-reported accessibility, continuity, and coordination of primary health care. Ann Fam Med 2008;6:116-23.

10. Hutchison B. From Hall to now: reflections on Canadian Medicare from a primary care perspective. Emmett Hall Memorial Lecture. Vancouver: Canadian Association for Health Services and Policy Research (CAHSPR), 2013.

11. Macinko J, Starfield B, Shi L. The contribution of primary care systems to health outcomes within Organization for Economic Cooperation and Development (OECD) countries, 1970-1998. Health Serv Res 2003;38:831-65.

12. Contandriopoulos D, Perroux M, Cockenpot A, et al. Analytical typology of multiprofessional primary care models. BMC Fam Pract 2018;19:44.

13. Poghosyan L, Norful AA, Liu J, et al. Nurse practitioner practice environments in primary care and quality of care for chronic diseases. Med Care 2018;56:1-97.

14. Poghosyan L, Liu J, Norful AA. Nurse practitioners as primary care providers with their own patient panels and organizational structures: a cross-sectional study. Int J Nurs Stud 2017;74:1-7.

15. Auerbach DI, Staiger DO, Buerhaus PI. Growing Ranks of Advanced Practice Clinicians - Implications for the Physician Workforce. N Engl J Med 2018;378:2358-60.

16. Wagner EH, Flinter M, Hsu C, et al. Effective team-based primary care: observations from innovative practices. BMC Fam Pract 2017;18:13.

17. Contandriopoulos D, Duhoux A, Roy B, et al. Integrated Primary Care Teams (IPCT) pilot project in Quebec: a protocol paper. BMJ Open 2015;5:e010559.
18. Donaldson $\mathrm{L}$. The normal science of structural contingency theory. In: Clegg SR, Hardy C, Nord WR, eds. Handbook of Organization Studies. Thousand Oaks, CA: SAGE Publications, 1996:57-76.

19. Norful AA, de Jacq K, Carlino R, et al. Nurse Practitioner-Physician Comanagement: A Theoretical Model to Alleviate Primary Care Strain. Ann Fam Med 2018;16:250-6.

20. Fayol H. General and industrial management. London: Pitman, 1949.

21. Taylor FW. The principles of scientific management. New York: Harper, 1911.

22. Hasenfeld Y. Human services as complex organizations. 2nd ed. Thousand Oaks, CA: Sage Publications, 2010

23. Abernethy MA, Stoelwinder JU. The role of professional control in the management of complex organizations. Accounting, Organizations and Society 1995;20:1-17.

24. Mintzberg $\mathrm{H}$. The structuring of organizations: a synthesis of the research. Englewood Cliffs, N.J.: Prentice-Hall, 1979.

25. Hall RH, Johnson NJ, Haas JE. Organizational size, complexity, and formalization. Am Sociol Rev 1967;32:903-12.

26. Fredrickson JW. The strategic decision process and organizational structure. Acad Manage Rev 1986;11:280-97.

27. Hage J, Aiken M, technology R. social structure and organizational goals. Administrative Science Quarterly 1969;14:368-79.

28. Dalton DR, Todor WD, Spendolini MJ, et al. Organization structure and performance: a critical review. Acad Manage Rev 1980;5:49-64.

29. Rizzo JR, House RJ, Lirtzman SI. Role conflict and ambiguity in complex organizations. Adm Sci Q 1970;15:150-63.

30. Meyer JW, Rowan B. Institutionalized organizations: formal structure as myth and ceremony. Am J Sociol 1977;83:340-63.

31. Powell WW, DiMaggio PJ. The New institutionalism in organizational analysis. Chicago: University of Chicago Press, 1991.

32. Scott WR. Organizations: Rational, Natural and Open Systems. Upper Saddle River, NJ: Prentice Hall, 2003.

33. Liberati EG. Separating, replacing, intersecting: the influence of context on the construction of the medical-nursing boundary. Soc Sci Med 2017;172:135-43.

34. Abbott A. The System of Professions. Chicago, IL: The University of Chicago Press, 1988.

35. Heale R, Butcher M. Canada's First Nurse Practitioner -Led Clinic : A Case Study in Healthcare Innovation. Nurs Leadersh 2010;23:21-9.

36. Martínez-González NA, Djalali S, Tandjung R, et al. Substitution of physicians by nurses in primary care: a systematic review and metaanalysis. BMC Health Serv Res 2014;14:214.

37. Wong FK, Chung LC. Establishing a definition for a nurse-led clinic: structure, process, and outcome. J Adv Nurs 2006;53:358-69.

38. Patton MQ. Developmental Evaluation: Applying Complexity Concepts to Enhance Innovation and Use. New-York, NY: The Guilford Press, 2011.

39. Quinn Patton M. Developmental evaluation. Eval Pract 1994;15:311-9.

40. Adamson BJ, Kenny DT, Wilson-Barnett J. The impact of perceived medical dominance on the workplace satisfaction of Australian and British nurses. J Adv Nurs 1995;21:172-83.

41. Hojat M, Fields SK, Veloski JJ, et al. Psychometric properties of an attitude scale measuring physician-nurse collaboration. Eval Health Prof 1999;22:208-20.

42. Hardy C, Palmer I, Phillips N. Discourse as a Strategic Resource. Human Relations 2000;53:1227-48.

43. Chouliaraki L, Fairclough N. Discourse in late modernity: rethinking critical discourse analysis. Edinburgh: Edinburgh University Press, 1999.

44. Moch MK, Fields WC. Developing a content analysis for interpreting langage use in organizations. Research in the sociology of organizations 1985;4:81-126.

45. Kirsch C, Bernier B. Le sens du discours écrit: propos méthodologiques à partir de deux recherches. Culture 1988;8:35-47.

46. Bourdieu P. Ce que parler veut dire: l'économie des échanges linguistiques. Paris: Fayard, 1982.

47. Lawrence PR, Lorsch JW. Differentiation and integration in complex organizations. Adm Sci Q 1967;12:1-47.

48. American Medical Directors Association Ad Hoc Work Group on the Role of the Attending Physician and Advanced Practice Nurse. Collaborative and supervisory relationships between attending physicians and advanced practice nurses in long-term care facilities. Geriatr Nurs 2011;32:7-17.

49. Bush NJ, Watters T. The emerging role of the oncology nurse practitioner: a collaborative model within the private practice setting. Oncol Nurs Forum 2001;28:1425-31.

50. DiCenso A, Matthews S. Report of the Nurse Practitioner Integration Task Team submitted to the Ontario Minister of Health and LongTerm Care. Toronto, ON: Ministry of Health and Long-Term Care, 2007 
51. Poghosyan L, Aiken LH. Maximizing nurse practitioners' contributions to primary care through organizational changes. $J$ Ambul Care Manage 2015;38:109-17.

52. Wranik WD, Haydt SM, Katz A, et al. Funding and remuneration of interdisciplinary primary care teams in Canada: a conceptual framework and application. BMC Health Serv Res 2017:17:1-12.

53. Heinemann GD, Schmitt MH, Farrell MP, et al. Development of an attitudes toward health care teams scale. Eval Health Prof 1999;22:123-42. 